\title{
Attention deficit hyperactivity disorder in children and adolescents
}

\author{
A J Flisher, S Hawkridge
}

\section{Introduction}

Attention deficit hyperactivity disorder (ADHD), although commonest in childhood and adolescence, can be diagnosed across the age span. The Diagnostic and Statistical Manual of Mental Disorders, Fourth Edition, Text Revision (DSM-IV-TR) ${ }^{[1]}$ defines the disorder using the core features of hyperactivity, impulsivity and/or inattention which are inappropriate for developmental stage.

The major aetiological influence for ADHD is genetic, which accounts for $80 \%$ of the variability of the disorder. ${ }^{[2,3]}$ However, the environment and environment-gene interactions are also significant. Examples of social factors associated with ADHD are low socioeconomic status, low parental education, family conflict and non-intact nuclear families, parental mental disorder, substance abuse, criminality, punitive parenting, severe early deprivation and institutional upbringing. ${ }^{[4]}$ Examples of pre-, peri- and postnatal environmental insults that are associated with ADHD include low birth weight, maternal prenatal smoking, stimulant and alcohol use, obstetric complications, head injury, epilepsy, HIV/AIDS, iron and zinc deficiency, lead exposure, and early and increased television exposure. ${ }^{[4]}$

The treatment guidelines that follow draw on and are broadly compatible with the following documents, which should be consulted for further information:

- Practice Parameter for the Assessment and Treatment of Children and Adolescents With Attention-Deficit Hyperactivity Disorder of the American Academy of Child and Adolescent Psychiatry ${ }^{[5]}$

- Treatment guidelines of the National Institute for Health and Clinical Excellence (NICE) on methylphenidate, atomoxetine and dexamphetamine for attention deficit hyperactivity disorder (ADHD) in children and adolescents ${ }^{[6]}$

- European Guidelines for hyperkinetic disorder ${ }^{[7,8]}$

- Flisher et al. ${ }^{[9]}$ 'Packages of care for attention deficit hyperactivity disorder in low- and middle-income countries'.

\section{Diagnosis and clinical characteristics 2.1 Screening}

Screening for ADHD should be included in the psychiatric assessment of every patient, regardless of the main complaint. Questions about the core symptoms of ADHD should be asked to assess whether a diagnosis of ADHD is possibly applicable. If it is, then a complete assessment should be performed. The diagnosis is not appropriate if the symptoms occur exclusively during the course of a pervasive developmental disorder, schizophrenia, or another psychotic disorder, or are better accounted for by another psychiatric disorder. The diagnosis is also inappropriate if the symptoms are better accounted for by a substance use disorder (e.g. intoxication) or a general medical condition such as allergic conditions, sensory deficits, chronic infections, etc. Collateral information is essential, particularly with regard to performance at school.

There are two types of instrument available to detect (and diagnose) ADHD: clinical diagnostic interviews and rating scales. The Diagnostic Interview Schedule for Children (DISC-IV) has been assessed for use in South Africa. ${ }^{[10]}$ No specific rating scales for ADHD have been assessed in South Africa, but the Conner's Parent Rating Scale - Revised (CPRS-R) and the Conner's Teacher Rating Scale Revised (CTRS-R) ${ }^{[11]}$ are often used. However, the AD/HD Rating Scale-IV ${ }^{[12]}$ may be a better choice since the symptoms correspond directly to those in DSM-IV-TR.

\subsection{Evaluation}

Evaluation of the child or adolescent for ADHD should consist of clinical interviews with the caregiver and patient, obtaining information about the patient's school or day-care functioning, evaluation for comorbid psychiatric disorders, and review of the patient's medical, developmental, social and family history.

Clinical observation of the patient should occur more than once and if possible in more than one setting. School visits are recommended if there is a discrepancy between reports from home and school or if there is uncertainty about the diagnosis. If possible, school observations should be carried out by a clinician other than those known to the child.

An important initial step is to determine whether the child or adolescent fulfils the criteria for the diagnosis. This involves establishing whether each of the 18 symptoms is present; confirming that at least 6 of the inattentive or at least 6 of the hyperactivity/ impulsivity cluster are present more days than not; that there is a chronic course; that onset was before the age of 7 years; and that the symptoms are associated with functional impairment. There is a significant risk of misdiagnosing other psychiatric disorders as ADHD, particularly anxiety disorders, and it is important to explore the child's emotional symptoms (e.g. worry, anxiety, beliefs, somatic symptoms, etc.). Oppositional defiant disorder can also be misdiagnosed as ADHD, because of some overlap of symptoms.

It is equally necessary to establish whether comorbid disorders are present, the most common of which are oppositional defiant disorder and conduct disorder. Other important comorbid disorders include intellectual disability, depression, mania, anxiety disorders, tic disorders, substance abuse, and specific learning disability. In some cases symptoms of inattention and hyperactivity may be attributable to another disorder, and not ADHD. In other cases, a child may suffer from symptoms that do meet criteria for a disorder and are a direct consequence of ADHD. For example, a child may become depressed owing to social isolation or academic difficulties that are caused by ADHD. Finally, a child may satisfy the criteria for ADHD as well as 
another disorder. In such cases, treatment may be necessary for both disorders.

It is generally helpful to use a symptom scale, which can be completed by the clinician, teacher and parent, although a diagnosis should never be made only on the basis of such a scale. However, extreme scores should raise the index of suspicion. The main use of symptom scales is to monitor response to treatment.

It is important to assess the patient's family, as untreated ADHD in family members is a common finding. Providing treatment for family members can often have collateral benefits for the child or adolescent who has been referred. Children or adolescents with ADHD function better in structured environments, and an unstructured environment may be the result of parental ADHD.

\subsection{Clinical presentation}

A thorough mental status and physical examination of the patient is mandatory, during which particular care should be taken to assess whether other psychiatric disorders are present. It is important to note that at the first session, hyperactivity may not be particularly evident as the child may initially be inhibited or making an extra effort to behave well for the occasion, so it is useful to see the child more than once before making a diagnosis of ADHD. Girls in particular may present with predominantly inattentive symptoms and this may not be obvious in the consulting room unless specifically sought.

\section{Assessment}

\subsection{Laboratory or neurological testing}

If there is a medical disorder that can account for the symptoms of ADHD, this will almost always be evident from the history and physical examination. Examples of such disorders include brain injury, hyperthyroidism, encephalopathies, lead poisoning and fetal alcohol syndrome. In the absence of any specific indications of such pathology, laboratory or neurological testing is not indicated. However, there is a significant comorbidity of epilepsy with ADHD, and a high index of suspicion should be maintained, bearing in mind that a normal electroencephalogram (EEG) is not necessarily proof of the absence of a seizure disorder, and an evaluation by a neurologist may be helpful.

\subsection{Psychometric testing}

Psychological and neuropsychological tests are not diagnostic for $\mathrm{ADHD}$, but should be performed if the patient's history suggests low general cognitive ability or low achievement in language or mathematics relative to the patient's intellectual ability. It is common for those with ADHD to perform poorly on testing owing to poor concentration and impulsivity. This should be taken into account when interpreting results, and results should be regarded as conclusive only once the child is being successfully treated for ADHD.

It is not uncommon for children and adolescents with ADHD to have evidence of poor educational attainment. The clinician needs to assess whether the low level of educational attainment is entirely due to the ADHD; whether the symptoms attributable to ADHD are in fact the result of other learning or language problems; or whether ADHD and other problems are both present. In the South African context, tests or norms may not be available for all language and cultural groups, and interpretation of the findings should take this into account.

\section{Treatment}

\subsection{Treatment goals}

The aim of treating ADHD is to optimise the child's cognitive, social and emotional functioning so as to prevent the development of secondary emotional distress or psychiatric disorders, and allow the child to reach his/her full developmental potential. Symptoms of inattentiveness and hyperactivity/impulsivity should be targeted, but the child's overall functioning, including family and social relationships, leisure activities and self-esteem must also receive attention.

\subsection{General aspects of treatment}

It is important to develop a treatment plan once the diagnosis has been established. This plan can be changed in the light of responses to treatment options, the emergence of other issues that need to be addressed and changing family circumstances. The treatment plan should always include psycho-education of the patient and their caregivers and educators. It should include information about ADHD, helping parents and teachers to anticipate developmental challenges that are difficult for children and adolescents with ADHD, and general advice to improve the child's academic, social and behavioural functioning.

\subsection{Acute treatment}

In recent years, much research attention has been focused on the relative indications for various forms of management, from which a considerable degree of consensus has emerged. Based on the existing guidelines, we propose that behavioural treatments may be applicable as first-line treatment if:

- The ADHD is mild or (perhaps) moderate, with minimal impairment

- The diagnosis is uncertain

- The parents (or patient) reject medication as a treatment option

- There is disagreement between key stakeholders about the diagnosis

- There are no comorbid diagnoses or significant life stressors

- An urgent response is not required.

However, if the patient's response to behavioural interventions is suboptimal, in that there is still significant impairment, relationships are still affected, or development is held back, then a trial of medication should be instituted. For severe cases, or where the above circumstances do not pertain, a trial of medication is appropriate as a first line of intervention.

Further steps following the initiation of treatment are indicated in the algorithm (Fig. 1).

Comorbid psychiatric conditions should be independently addressed by an appropriate child and adolescent mental health professional.

\subsection{Maintenance treatment}

Children who are taking stimulant medication require monthly prescriptions (repeat prescriptions are not permitted for Schedule 6 drugs) and at least 6-monthly reviews by a psychiatrist. At each review the following should be recorded: 


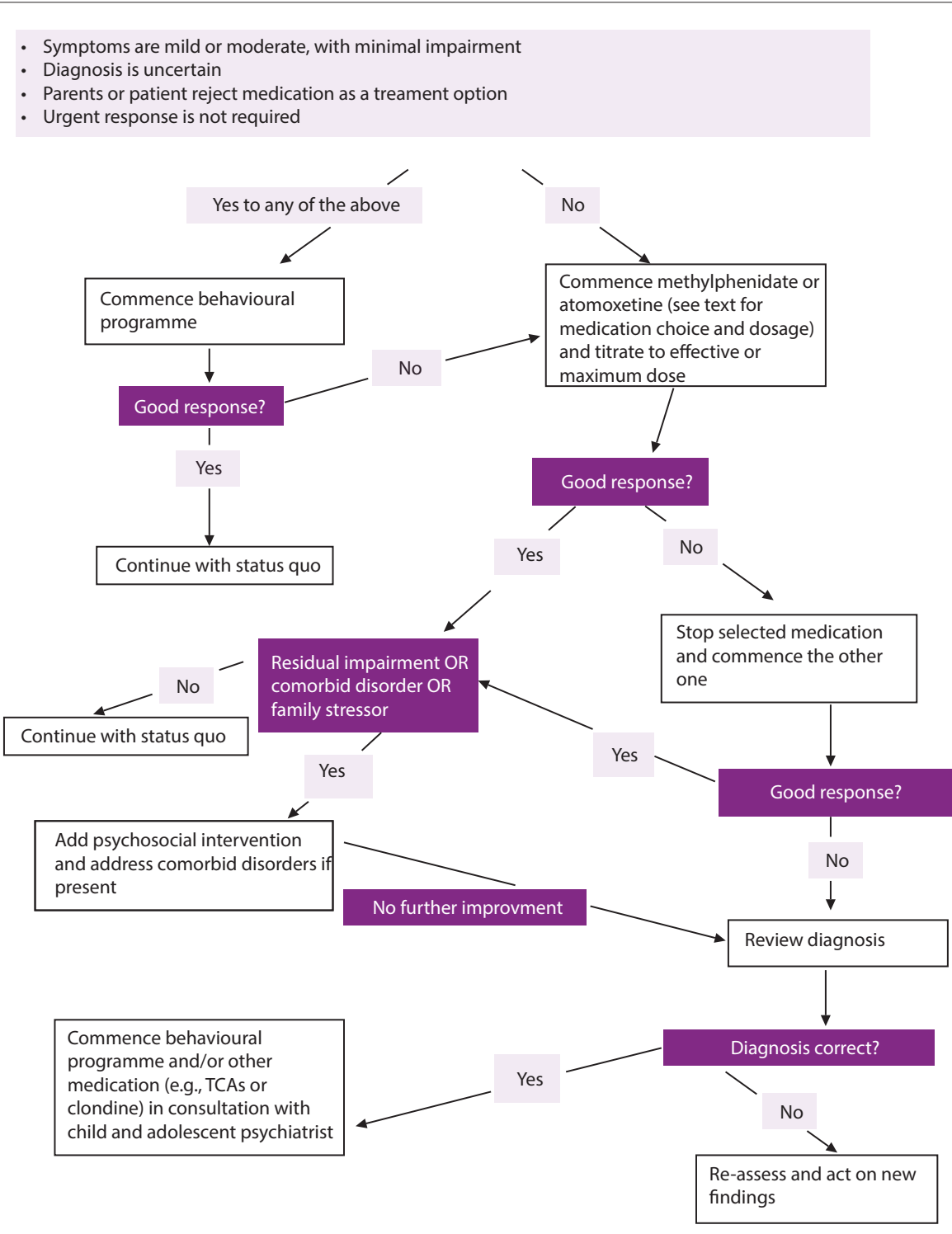

Fig. 1. Treatment algorithm (TCAs - tricyclic antidepressants).

- Weight and height (recorded on a percentile chart)

- Reported adverse effects

- Efficacy (school and parent reports)

- Pulse rate and blood pressure.

Significant deviation from the child's usual percentile in terms of weight or height should provoke a review of dosage as well as the institution of a 'drug holiday' of at least 4 weeks, preferably during school holidays, so that catch-up growth can occur. Should this not happen, alternative medication or non-pharmacological treatment should be considered. Should failure to gain weight continue, consultation with a paediatrician should be requested. later and if persistent, stimulant medication should be withdrawn. Should the abnormal cardiovascular parameters persist without medication, a referral to a paediatrician or physician is mandatory. If there is a return to normal parameters, then alternative medication or non-pharmacological treatments should be instituted.

If a patient with ADHD has been symptom-free for at least 1 year, the clinician may consider stopping the medication. Clues that this may be appropriate include: not needing to adjust the dose despite an increase in growth; the lack of obvious symptoms when a dose is accidentally omitted; and observations of adequate concentration during drug holidays. Medication can be tapered or stopped at a time when stressors are absent or minimal, and once the school term is under way. It is important to obtain feedback from the patient, the parents and the educators about the effect of this change. If symptoms recur, it is necessary to reinstitute treatment immediately.

\subsection{Pharmacological treatment}

Pharmacotherapy of ADHD with psychostimulants is one of the best established and most consistently demonstrated effective treatments in psychiatric medicine. There is consistent evidence of effect in domains such as symptomatic improvement; enhanced cognitive, social, family and academic functioning; and improvement of nondiagnostic symptoms frequently associated with the condition such as irritability, aggressive outbursts and difficulties with fine motor co-ordination. ${ }^{[13-16]}$ Significant and clinically substantial effect sizes $(0.8-1.1)$ of methylphenidate have been reported from analyses of clinical studies. ${ }^{[8]}$ Results from the most comprehensive study of ADHD treatment (the Multimodal Treatment Study of Children with ADHD (MTA)) strongly support the use of methylphenidate as a treatment for young people (school age into adolescence) with ADHD. ${ }^{[17]}$

Dosing should be started as low as possible (usually $5-10 \mathrm{mg}$ ) in a morning dose of a short-acting formulation. The effect of shortacting methylphenidate lasts approximately 3 hours, and the teacher should be able to give a report as to the efficacy in the first few hours of school. Should this be ineffective, an increased dose (10 - $20 \mathrm{mg})$ should be 
considered. Initial dosing should not exceed $1 \mathrm{mg} / \mathrm{kg} /$ day. If a response is obtained, the dose can be repeated at about $11 \mathrm{~h} 00$, and the outcome documented. Conversion to a long-acting formulation should then be considered. Should insufficient response to an optimal dose be seen, the protocol for partial and non-responders should be followed. Doses greater than $1 \mathrm{mg} / \mathrm{kg} /$ day may occasionally be necessary, but should only be considered in consultation with a child and adolescent psychiatrist.

The preferred formulation of methylphenidate is long-acting and removes the necessity for mid-morning doses, which are potentially embarrassing for the child and have become difficult owing to the paucity of school-based medical services and legislation prohibiting the possession or dispensing of scheduled medication by unqualified individuals. Long-acting formulations have the additional benefit of maintaining blood levels of methylphenidate, giving more even efficacy. Adverse effects tend to be less troublesome, and adherence to medication is enhanced. Additional doses of short-acting formulation may be required for specific times of the day, either before the morning dose of long-acting formulation takes effect or to cover a possible afternoon period of rebound hyperactivity as the effect wears off.

Adverse effects of methylphenidate include headache, insomnia, gastric discomfort and decreased appetite (with concomitant weight loss or failure to gain weight appropriately). Anxiety and depression may be worsened by methylphenidate, prompting many clinicians to choose alternative medications for children with comorbid anxiety or mood disorders. Methylphenidate carries a Food and Drug Administration black box warning in respect of a slightly increased danger of sudden death. A family history of cardiovascular disease or sudden death, or an individual history of unexplained fainting or cardiovascular disorders, should cause the treating physician to refer the child to a cardiologist for an opinion before instituting psychostimulant therapy. In the absence of these risk factors, a baseline electrocardiograph is not regarded as mandatory by most authorities.

Children and adolescents with tic disorders may experience worsening of tics on methylphenidate. Some will consider the advantage of their improved school functioning worth the increase in tics; others should be offered other treatment options. Patients with mania or psychosis should not be given methylphenidate. Methylphenidate has been shown to protect against substance use disorders, but for patients with comorbid substance use disorders, alternative medications should be considered, although physical dependence is not a documented risk.

Other medications have also demonstrated positive therapeutic effects in ADHD across the life span, from primary school age to adulthood. ${ }^{[6]}$ The most intensively studied of these is atomoxetine, a noradrenergic reuptake inhibitor. ${ }^{[18-20]}$ Even though treatment effect sizes are less than for methylphenidate, it can be considered the agent of first choice for patients with an active substance abuse problem, comorbid anxiety, or tics; or if the patient experiences intolerable side-effects to methylphenidate, or if the patient (or caregiver) prefers this agent. Dosing of atomoxetine starts at $0.5 \mathrm{mg} / \mathrm{kg} / \mathrm{day}$ and can be titrated upwards at minimum 3-day intervals to $1.2 \mathrm{mg} / \mathrm{kg} /$ day. Adverse effects tend to be mild but severe liver damage can occur, and cardiovascular monitoring should be routine. Atomoxetine has been included in the group of drugs potentially causing an increase in suicidality in children and adolescents, so informed consent and appropriate psycho-education of patient and caregivers is mandatory.

Less evidence exists for the tricyclic antidepressants (desipramine and imipramine), buproprion and clonidine. ${ }^{[21]}$ These medications may be considered to be second-line pharmacotherapy alternatives because of the proportionally smaller research data base, shorter timeline of clinical experience, a potentially greater frequency of medically significant side-effects and greater expense compared with methylphenidate. ${ }^{[20]}$

\subsection{Non-pharmacological treatment}

Behavioural programmes are the first line of treatment in very young children, and in those whose difficulties are mild and where immediate relief is not required. Such programmes can be delivered by non-medical personnel such as mental health nurses or psychologists, provided adequate training has been done. Parent support groups for ADHD may be helpful in the sourcing of such resources.

The successful behavioural programmes ${ }^{[22]}$ consist of between 10 and 20 sessions of $1-2$ hours each in which parents are given information about the nature of ADHD and learn to:

- Attend more carefully to their child's behaviour and to notice when their child does or does not comply with requests

- Understand the principles and implementation of behaviour management

- Use time out effectively

- Use a daily school report card

- Anticipate future difficulties.

A systematic literature review ${ }^{[23]}$ and a meta-analysis investigating 174 ADHD treatment studies concluded that behavioural treatment is highly effective. ${ }^{[24]}$

\subsection{Special populations}

Data from preschool-age populations are sparse, but those available support use of psychostimulants for the treatment of $\mathrm{ADHD}^{[18]}$ with the caveat that psychosocial modalities, especially parent training, should be the initial interventions of first choice. ${ }^{[25]}$ Should consideration be given to treating a preschool child with a stimulant drug, consultation with a child and adolescent psychiatrist should be obtained where possible.

The treatment of adolescents with ADHD is the same as that for children, but special attention must be given to the developmental trajectory of adolescents. Increasing weight will usually require an increased dose. Sporting activities at a high level (where drug testing is carried out) may require a switch to non-stimulant medication, and social changes make it imperative that substance use disorders should be specifically asked about and managed appropriately. Some adolescents may find themselves in a tertiary education environment and others may be in the workplace before they turn 18. These individuals should be managed as adults. Symptoms persist into adulthood in at least one-third of children with ADHD, and ADHD is increasingly diagnosed in adults. Diagnostic criteria and management are modified for the different environment and obligations of adults, and separate guidelines are required.

\subsection{Managing partial and non-responders}

Partial response may be dose-related, or there may be undiagnosed comorbid conditions which are limiting response. Should there be no 


\section{fre

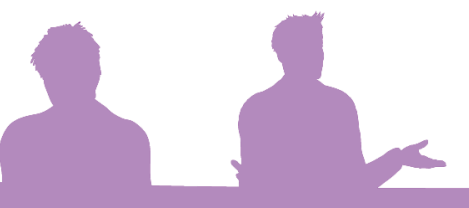

response to therapeutic doses of pharmacological treatment or parent training/behavioural programmes, the diagnosis should be reviewed. Pervasive developmental disorders, mood disorders and anxiety disorders are frequently misdiagnosed as $\mathrm{ADHD}$, and require very different management. An evaluation by, or at least consultation with, a child and adolescent psychiatrist should be obtained.

In ADHD with severe behavioural difficulties, atypical antipsychotic drugs are sometimes prescribed for impulsive aggression or temper outbursts. This should not be done without a complete review of the family and school environments, possible stressors, adequate psychosocial intervention and a clear risk-benefit evaluation of the potential adverse effects of the drug considered. The involvement of child mental health professionals is advised prior to such a step being taken.

\section{Summary points}

- The most important aspect of treating ADHD is an accurate diagnosis. Making the incorrect diagnosis may render treatment futile or even harmful.

- In mild to moderate ADHD, first-line treatment is a behavioural programme with or without pharmacological modalities.

- In moderate to severe ADHD, first-line treatment is a pharmacological agent plus a behavioural programme.

- Family education and intervention where necessary is mandatory.

References

1. American Psychiatric Association. Diagnostic and Statistical Manual of Mental Disorders: Fourth Edition, Text Revision (DSM-IV-TR). Washington DC: American Psychiatric Association, 2000

2. Biederman J. Attention-deficit/hyperactivity disorder: A selective overview. Biol Psychiatry 2005;57:1215-1220. [http://dx.doi.org/10.1016/j.biopsych.2004.10.020]

3. Biederman J, Faraone SV. Attention deficit hyperactivity disorder. Lancet 2005;366:237-248. [http://dx.doi.org/10.1016/S0140-6736(05)66915-2]

4. Patel V, Lund C, Hatherill S, et al. Social determinants of mental disorders. In: Blas E, Sivasankara Kurup A, eds. Priority Public Health Conditions: From Learning to Action on Social Determinants of Health. Geneva: World Health Organization (in press).

5. American Academy of Child and Adolescent Psychiatry. Practice parameter for the assessment and treatment of children and adolescents with attention-deficit/hyperactivity disorder. J Am Acad Child Adolesc Psychiatry 2007;46:894-921.

6. National Institute for Health and Clinical Excellence (NICE). Methylphenidate, atomoxetine and dexamphetamine for attention deficit hyperactivity disorder $(\mathrm{AD} / \mathrm{HD})$ in children and adolescents. Technology appraisal 98. London: NICE, 2008.
7. Taylor E, Sergeant J, Doepfner M, et al. Clinical guidelines for hyperkinetic disorder. Eur Child Adolesc Psychiatry 1998;7:184-200. [http://dx.doi.org/10.1007/s007870050067]

8. Taylor E, Dopfner M, Sergeant J, et al. European clinical guidelines for hyperkinetic disorde - first upgrade. Eur Child Adolesc Psychiatry 2004;13:17-130. [http://dx.doi.org/10.1007/ s00787-004-1002-x]

9. Flisher AJ, Sorsdahl K, Hatherill S, Chehil S. Packages of care for attention-deficit hyperactivity disorder in low- and middle-income countries. PLoS Medicine 2010;7:e1000235. [http://dx.doi.org/10.1371/journal.pmed.1000235]

10. Flisher AJ, Lund C, Sorsdahl K, Robertson B. Test-retest reliability of the Xhosa version of the Diagnostic Interview Schedule for Children (abstract). Journal of Child and Adolescent Mental Health 2009;21:88. [http://dx.doi.org/10.1111/j.1365-2214.2010.01195.x]

11. Conners CK. Conners Rating Scales - Revised: Technical Manual. Toronto: Multi-Health Systems, 1997.

12. DuPaul GJ, Power TJ, Anastopoulos AD, Reid R. AD/HD rating scales - IV: Checklists, Norms and Clinical Interpretation. New York: Guilford, 1998.

13. Vitiello B. Methylphenidate in the treatment of children with attention-deficit hyperactivity disorder. CMAJ 2001;165:1505-1506.

14. Banaschewski T, Coghill D, Santosh P, et al. Long-acting medications for the hyperkinetic disorders: A systematic review and European treatment guidelines. Eur Child Adolesc Psychiatry 2006;15:476-495. [http://dx.doi.org/10.1007/s00787-006-0549-0]

15. Karande S. Attention deficit hyperactivity disorder - a review for family physicians. Indian J Med Sci 2005;59:546-555. [http://dx.doi.org/10.4103/0019-5359.19200]

16. Kratochvil CJ, Egger HL, Greenhill LL, McGough JJ. Pharmacological management of preschool AD/HD. J Am Acad Child Adolesc Psychiatry 2006;45:115-118. [http://dx.doi. org/10.1097/01.chi.0000186451.49579.0a]

17. The MTA Cooperative Group. A 14-month randomized clinical trial of treatment strategies for attention deficit/hyperactivity disorder (AD/HD). Arch Gen Psychiatry 1999;56:10731086. [http://dx.doi.org/10.1001/archpsyc.56.12.1073]

18. Michelson D, Allen AJ, Busner J, et al. Once-daily atomoxetine treatment for children and adolescents with attention deficit hyperactivity disorder: A randomized, placebo-controlled study. Am J Psychiatry 2002;159:1896-1901. [http://dx.doi.org/10.1176/appi.ajp.159.11.1896]

19. Michelson D, Buitelaar JK, Danckaerts M, et al. Relapse prevention in pediatric patients with $\mathrm{AD} / \mathrm{HD}$ treated with atomoxetine: A randomized, double-blind, placebo-controlled study. J Am Acad Child Adolesc Psychiatry 2004;43:896-904. [http://dx.doi.org/10.1097/01. chi.0000125089.35109.81]

20. Michelson D, Faries D, Wernicke J, et al. Atomoxetine in the treatment of children and adolescents with attention-deficit/hyperactivity disorder: A randomized, placebocontrolled, dose-response study. Pediatrics 2001;108:1-9. [http://dx.doi.org/10.1542/ peds.108.5.e83]

21. Pliszka SR. Non-stimulant treatment of attention-deficit/hyperactivity disorder. CNS Spectrums 2003;8:253-258.

22. Smith BH, Barkley RA, Shapiro CJ. Attention deficit hyperactivity disorder. In: Mash EJ, Barkley RA, eds. Treatment of Childhood Disorders, 3rd ed. New York: Guilford Press, 2006.

23. Chronis AM, Jones HA, Raggi VL. Evidence-based psychosocial treatments for children and adolescents with attention-deficit/hyperactivity disorder. Clin Psychol Rev 2006;26:486-502. [http://dx.doi.org/10.1016/j.cpr.2006.01.002]

24. Fabiano G, Pelham WE, Coles EK, et al. A meta-analysis of behavioural treatments for attention-deficit/hyperactivity disorder. Clin Psychol Rev 2009;29:129-140. [http://dx.doi. org/10.1016/j.cpr.2008.11.001]

25. Taylor E, Döpfner M, Sergeant J, et al. European clinical guidelines for hyperkinetic disorder - first upgrade. Eur Child Adolesc Psychiatry 2004;13:17-30. [http://dx.doi.org/10.1007/ s00787-004-1002-x] 